\title{
The Effectiveness of Using Discrete Event Simulation to Optimize the Quality of Service of Outpatient in Iraq: A Case Study
}

\author{
${ }^{1}$ Maysoon A. Mohammed*, ${ }^{1}$ Shaymaa Kadhim Mohsin, ${ }^{1}$ Sarah Jasim Mohammed \\ ${ }^{1}$ University of Technology, Baghdad - Iraq
}

\section{Article information}

\section{Article history:}

Received: March, 26, 2021

Accepted: April, 25, 2021

Available online: June, 14, 2021

Keywords:

Discrete event simulation,

Outpatient clinic,

Dentists,

Arena software

*Corresponding Author:

Maysoon A. Mohammed

maysoon.a.mohammed@uotechnology.e du.iq

DOI:

https://doi.org/10.53523/ijoirVol8I1ID1

\begin{abstract}
One of the most important healthcare institutions in Iraq is Outpatient clinic that requires a lot of thinking to improve the way to provide services and the nature of care. Outpatient clinics are increasingly keen to meet the needs of care, and this has been recognized as a fundamental issue related to service quality. Therefore, many researchers in various fields have taken this matter as a basis for their research, as it is considered a rich material for research due to the problems these institutions contain. The most important problems faced by outpatient clinics are the waiting time and the insufficient number of clinic staff to perform the various tasks. The aim of this paper is to reduce the waiting time by building a model for the clinic environment, especially dental clinics, and trying to benefit from all the existing medical staff and exploit their experiences. Since the patient spends a long time between registration, returning to the doctor and finally the result or process that the doctor performs, building such a model might help in identifying and improving the problem. The simulation model built in this research for the clinic is based on modelling the discrete events inside the dental clinic using the Arena software. This form is used to assess the quality of services provided by dental outpatient clinics in Iraq.
\end{abstract}

\section{Introduction}

Simulation is one of the most powerful methodologies that have been investigated to study the problems and issues of some sophisticated service and manufacturing systems [1]. In general, models of simulation are designed to evaluate the changes in a system [2]. For instance, outpatient is one of the most important fields provided services in healthcare sectors. Simulation models are used to evaluate the level of services provided by the outpatient. The reason of using simulation models to evaluate such fields is the difficulty and cost of these fields. So, many necessary processes are constructed to optimize the services of outpatient are constructed. Thus, the outcomes of the simulation processes can be depended on to predict and forecast [3].

Healthcare sectors need to provide the best quality of services to patients [4]. This need may put healthcare sectors under a great pressure based on the requirements of the patients and their satisfaction of the services provided by 
such sectors. According to these requirements, healthcare sectors need to specify key care dimensions in terms of accessibility, waiting time and impact of care [5]. Outpatient clinics are characterized by rapid development, diversity of services and unpredicted cases [6]. The application of these characteristics of outpatient clinics requires efforts to improve the services at these clinics [7]. These characteristics are devoted to improving the performance and the quality services of the outpatient clinics by smoothing patient flow, utilizing resources effectively and formation a crisis cell to deal with critical cases [8]. Waiting time reduction is one of the most important principles which needs to be applied in the outpatient clinics to enhance the quality of services to satisfy the patients [9].

This paper investigates patients' flow in a dental outpatient clinic in local clinic in Iraq. The study aims to build a discrete-event simulation (DES) model in Arena software to show the environment in this clinic and how to improve it. A discrete-event simulation (DES) simulates an environment of a system as sequences of discrete events in time. Each event occurs at a particular instant in time and marks a change of state in the system [10]. The process followed in this clinic will be declared in a paradigm. The paradigm shows the procedure of flowing the patients to a local clinic including, registration, and decision for treating in the same clinic or sending to another specialist outpatient clinic. Information about the process of this study are collected through interviews with the patients to find out the problems in the clinic. The procedure in the clinic is defined by a paradigm, modelled and validated using an Arena DES Software. The DES model also is used to test the suggested enhancement scenario potential savings are reported. The suggested scenario aims to increase the resources and decrease the waiting time at the clinic.

\section{Literature Review}

In the past few decades, researchers suggested many methods in healthcare sector using modeling and simulation. The reason for the widespread using of modeling and simulation in the healthcare sector is availability, low costs and ease of use. Many of Simulation models are used in the mentioned sector as discrete event simulation (DES), agent-based simulation (ABS), dynamic system simulation (DSS) and so on. These models are designed to solve problems in different areas including healthcare sector. In healthcare, the models are used to simulate a certain purpose that focuses specifically on the issues that help to increase the efficiency of this organization, improve patient care, decrease costs and the ergonomic things for humans.

Due to the fact that healthcare sector is a sophisticated area where even a margin of error is not acceptable, the applications of simulation in the healthcare sector have become an essential element in determining the optimal way in reaching, serving, and treating patients. Researcher in [11], an arena software simulation has been used to test different alternatives for both the current and proposed project in urban outpatient. Their results show that, increasing the number of nurses responsible on the urgent patients serving over the Stat patients serving could improve the efficiency of the caring service. A review of combination of DES, ABS, and DES integration and ABS technique to solve the problem of modeling emergency waiting time has been made in [2]. This review summarizes and concludes the findings of the articles which used individual and combined techniques.

A real case study is used to describe the tools of facilitated DES supported with the experimentation and implementation stages in a DES study involving workshops with a group of stakeholders and the interactions at the workshops [12]. The paper puts forward an extensive line of proposed research in a bid to encourage other DES groups to enter this line of research. A new individual model of DES was built to reflect possible events and pathways of individuals invited to screening abdominal aortic aneurysm AAA [13]. Those not invited are using R programming language. The case of AAA screening highlights the benefits of DES, particularly in the context of screening studies. A research to present a systematic methodology for the development of DES model from process mapping model based on a complex collaborative healthcare service delivery processes which is Role Activity Diagram (RAD) is presented by [14]. A case study of magnetic resonance (MR) scanning process of radiology department in a large hospital is demonstrated for this methodology. A review paper is presented by [15] to compare and contrast the modeling methodologies related to emergency department (ED) crowding. The paper describes the assumptions, outlines and the limitations of each methodology in emergency department crowding. Researchers used a Model Driven Architecture which depends on an extension to Business Process Model and Notation (BPMN) and developed it to (BPMN4SIM) [16]. The result shows that BPMN4SIM is able to represent a complex business processes from elderly emergency care in a UK hospital. 


\section{Methodology and Proposed System}

Standard inputs to DES models include entities what flow through the system resources, locations, arrival rates, service times, and processing logic. Patients are the most common entities modeled in healthcare DES applications; however, lab specimens (e.g., blood), patient charts, and supplies are also exampling of entities. Resources process entities through the system and include human resources (e.g., nurses, physicians, lab technicians, and pharmacists) and equipment (e.g., MRI machines, lab analyzers, electrocardiograph machines). Locations are physical areas where resources process entities, such as treatment areas, registration, computer workstations, and lab and radiology facilities. Locations are often designed using facility layouts and scaled within the DES model. Arrival rates define the rate at which entities arrive at specific locations; examples might be the frequency of patients arriving at the ED or a scheduled clinic visit. Service times define the time needed for resources to process entities at set locations.

The classification of DES in this paper, the patients and record of patients are the entities, doctors and nurses are the resources, and the clinic is the location. A diagram is presented to show the process of the patients flow at the clinic as shown in Figure (1).

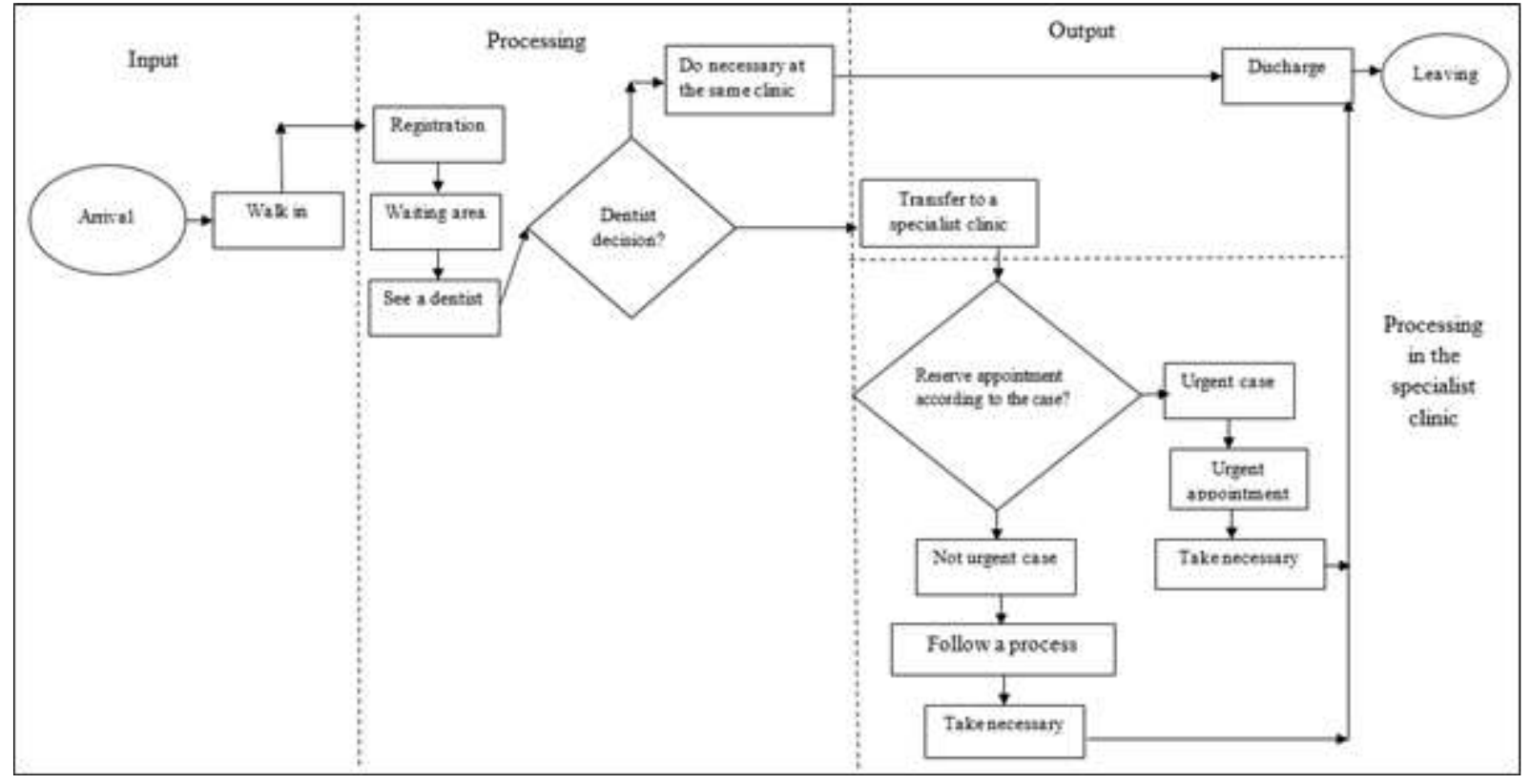

Figure (1). Diagram of patients flow at a clinic.

Figure 1 classified the patient flow to three-parts input, processing in local or specialist clinic, and output. The first part is the input process, where the patients arrive to the clinic walking in for registration which is the second part (processing part). The processing part contains three sections, registration, waiting area and seeing the doctor. After the patient takes his turn in queue and registers, he waits to see the doctor. The decision of the doctor depends on the case of the patient. If the equipment that need to treat the patient are available in the same clinic, so the doctor does the necessary and sends the patient for discharging which is the third part. Otherwise, if the patient needs special treatment which is unavailable in the same clinic, the doctor sends him to a specialist clinic. In the specialist clinic, there are two options to deal with the patient's case. If the patient's case is not urgent like teeth implant, the patient will take his turn and follow the schedule the procedure of the clinic. However, if the case is urgent like a toothache needs a root filling, the dentist deals with such cases immediately then sends the patient for discharging.

After visiting one of the outpatients in Iraq specifically in the capital (Baghdad), conducting interviews with the staff including (registration staff, nurses, some doctors, financial staff and pharmacy staff), we got the statistics shown in the tables below. Table (1) show us the daily work hours during week which include (open time, start time, registration, rest time and close time). 
Table (1). Daily work hours during week.

\begin{tabular}{|c|c|c|c|c|c|}
\hline Day week & Open time & Start time & Registration & Rest time & Close time \\
\hline Saturday & $7: 30$ & $8: 00$ & $8: 300$ & $1: 00-2: 00$ & $3: 00$ \\
\hline Sunday & $7: 30$ & $8: 00$ & $8: 300$ & $1: 00-2: 00$ & $3: 00$ \\
\hline Monday & $7: 30$ & $8: 00$ & $8: 300$ & $1: 00-2: 00$ & $3: 00$ \\
\hline Tuesday & $7: 30$ & $8: 00$ & $8: 300$ & $1: 00-2: 00$ & $3: 00$ \\
\hline Wednesday & $7: 30$ & $8: 00$ & $8: 300$ & $1: 00-2: 00$ & $3: 00$ \\
\hline Thursday & $7: 30$ & $8: 00$ & $8: 300$ & $12: 00-1: 00$ & $2: 00$ \\
\hline Friday & Holiday & Holiday & Holiday & Holiday & Holiday \\
\hline
\end{tabular}

From Table (1), we notice the difference between the open and registration time is one hour, this hour is for organizing the records, maintenance and clean works. Also, there is a difference between work hours on Thursday (one hour) because it is the end of week. These work hours are almost suitable in local and densely populated areas. The number of working employers in the outpatient is illustrated in Table (2).

Table (2). Number of working employers in the outpatient.

\begin{tabular}{|l|l|}
\hline Employers & \\
\hline Registration & 2 \\
\hline Nurses & 5 \\
\hline Doctors & 2 (esoteric), 3 (dentists), 2 (children), 2 (gynecology and infertility) \\
\hline Pharmacy & 2 \\
\hline Lab Employers & 2 \\
\hline Records employer & 1 \\
\hline Cleaners & 3 \\
\hline
\end{tabular}

The number of nurses in Table (2) above is 5, it is not enough compared with the number of the doctors in the outpatient. This issue needs to improve by increasing the number of nurses in the clinic. Also, the employer responsible on the records is only one. This causes delay and late in work, considering that the number of patients exceed 40 per day. Tables ( $3 \& 4$ ) illustrated the number of patients one day and the rooms of the doctors respectively.

Table (3). The number of patients for different cases.

\begin{tabular}{|c|c|c|c|c|c|c|}
\hline \multirow{2}{*}{ Hour } & \multirow{2}{*}{$\begin{array}{c}\text { approximate } \\
\text { Total } \\
\end{array}$} & \multicolumn{6}{|c|}{ approximate numbers } \\
\cline { 5 - 7 } & number & Dentist & Children & Esoteric & $\begin{array}{c}\text { Gynecology } \\
\text { and infertility }\end{array}$ & $\begin{array}{c}\text { Medical } \\
\text { analysis }\end{array}$ \\
\hline $8: 30-9: 30$ & 10 & 3 & 2 & 2 & 2 & 1 \\
\hline $9: 30-10: 30$ & 15 & 7 & 2 & 2 & 2 & 2 \\
\hline $10: 30-11: 30$ & 10 & 4 & 2 & 2 & 1 & 1 \\
\hline $11: 30-1: 00$ & 5 & 2 & 0 & 1 & 2 & 0 \\
\hline Total & 40 & 16 & 6 & 7 & 7 & 4 \\
\hline
\end{tabular}


Table (4). Rooms of the clinic.

\begin{tabular}{|l|l|}
\hline \multicolumn{1}{|c|}{ Room } & \\
\hline Dentist & 2 \\
\hline Children & 1 \\
\hline Esoteric & 1 \\
\hline Gynecology and infertility & 2 \\
\hline Labs & 1 \\
\hline Records & 1 \\
\hline x-rays & 1 \\
\hline Sonar & 1 \\
\hline Teeth x-rays & 1 \\
\hline
\end{tabular}

From Table (3), we notice that the number of flowing patients to the clinic in the early working hours is large and begins to decrease as the time approaches a rest time. After rest time, there is no registration so that doctors can see as many patients as possible.

Table (4) shows us that the rooms are not enough for the number of doctors, where each one must get one special check room for keeping the privacy of patients. This issue needs to improve by adding more rooms to the clinic. For example, there are three dentists, while there are only two rooms for them, the solution by either adding a new check chair or a new room.

The number of patients is large, some days exceeds 40 patients per day, so it is important to increase the number of nurses and rooms.

The focus of this research is the dentist issue and patients, where the number of patients who want to see the dentists is the largest in the clinic. The number of dentists is three and only two rooms, this causes load on the dentists, delay in work and overcrowded patients. Also, if the dentists could not accommodate all patients, this leads to either a patient wait his turn in the next day and will repeat all process again or go to another special clinic or special dentist which is very expensive.

Due to the information that we got from the interviews, the work of the third dentist is only rotating between two rooms, documenting the name of patients and describing the medication according to the instructions of the senior dentist. This is not a good thing as the experience of the third dentist and what he learned during his study will be wasted. And also, rotating between two rooms causes delay in work and disruption of patients.

So, there are many suggestions to benefit from the experience of the third dentist:

$>$ Increase the number of dentists which leads to 2 .

$>$ Increase the number of rooms.

Add one more check chair.

Increase the number of nurses.

Since these suggestions lead to spend too much money, and this is difficult for the administration of some clinics, so another solution must be found to solve this problem and benefit as much as possible from the experience of the third dentist and not waste time.

To benefit from the experience of the third dentist, we suggest that the third dentist examines patients and identify which case requires treatment inside the current clinic and which case requires referral to an outside clinic. This will make better use of time, accommodate the largest possible number of patients and reduce wasted time. 
Two DES models have been built to simulate the performance of the dentists at the current state and after the improvement. The first model is for the clinic at the current state, and the second model is after implementing our suggestion. The models have been built using Arena simulation software and illustrated in the figures below.

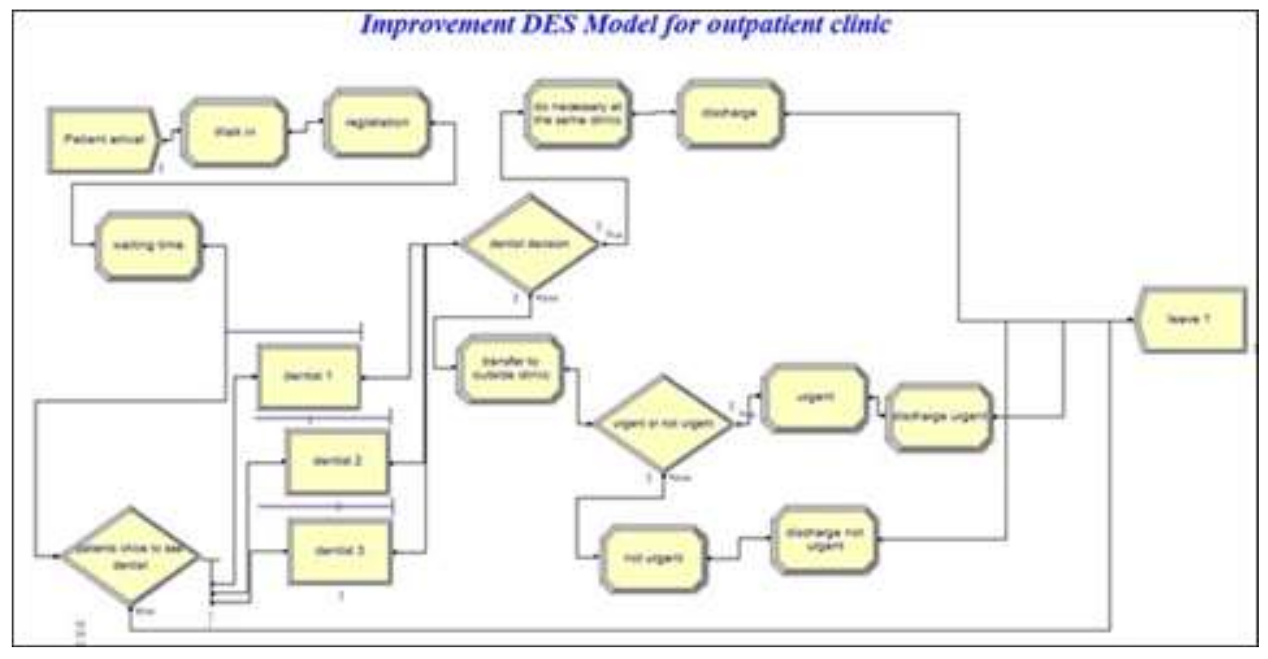

Figure (2). DES model for outpatient clinic at the current state.

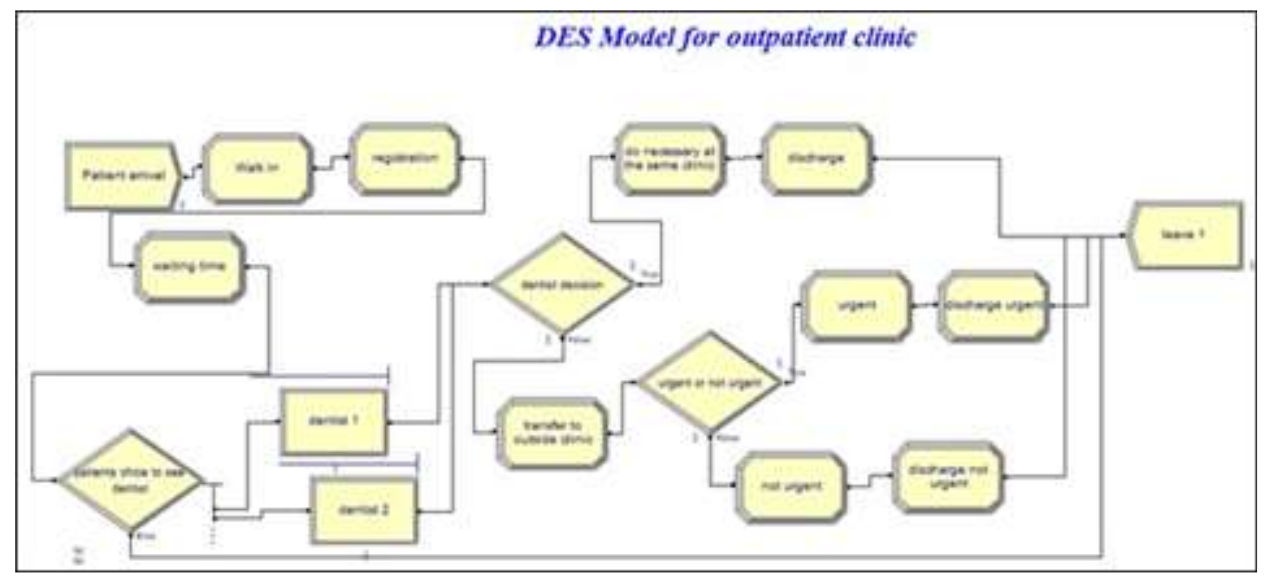

Figure (3). DES model for outpatient clinic after improvement.

Figure (2) shows us the model with to resources (which mean the dentists), while in Figure (3), the model using three resources. After executing the models in Figure ( $1 \& 2)$ we got the report results illustrated in Figure (4).

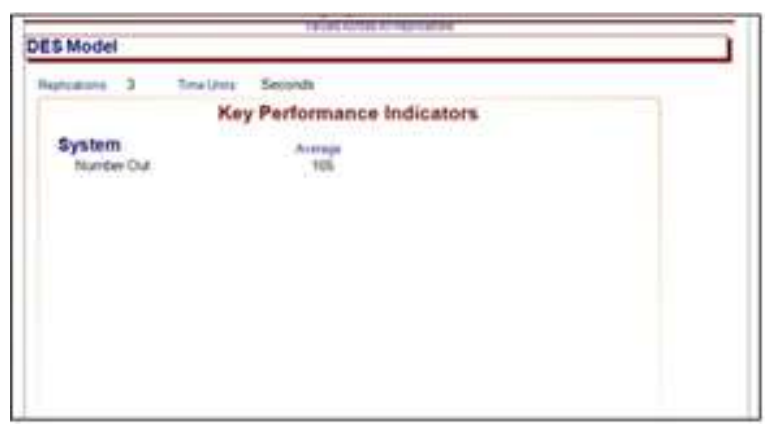

Figure (4-a). Category overview of model 1.

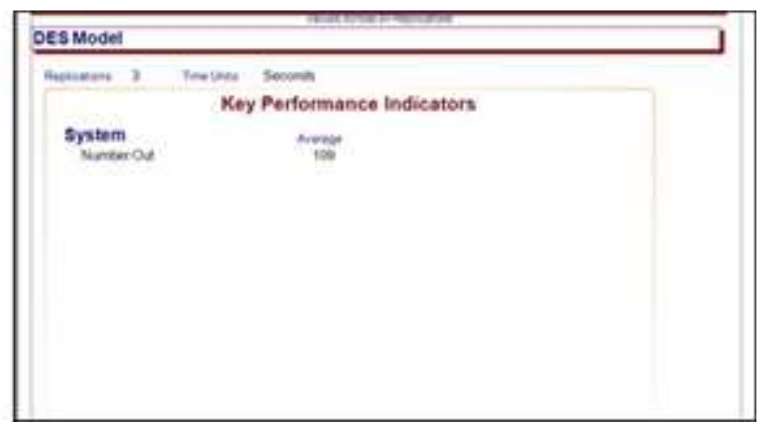

Figure (4-b). Category overview of model 2. 
In Figure ( 4 a \& b), we notice that, the average of number out of patients in model 2 is larger than in model 2 with 3 . This means that the number of resources leads to increase the number of patients.

\section{Results and Discussion}

To get better result we implement 3 replications for the two models, the results of the number of patients in to dentist and out from dentist for the 3 replications for model 1 and 2 have been presented in Figures (5, 6, 7, 8, 9, $\& 10)$, respectively.

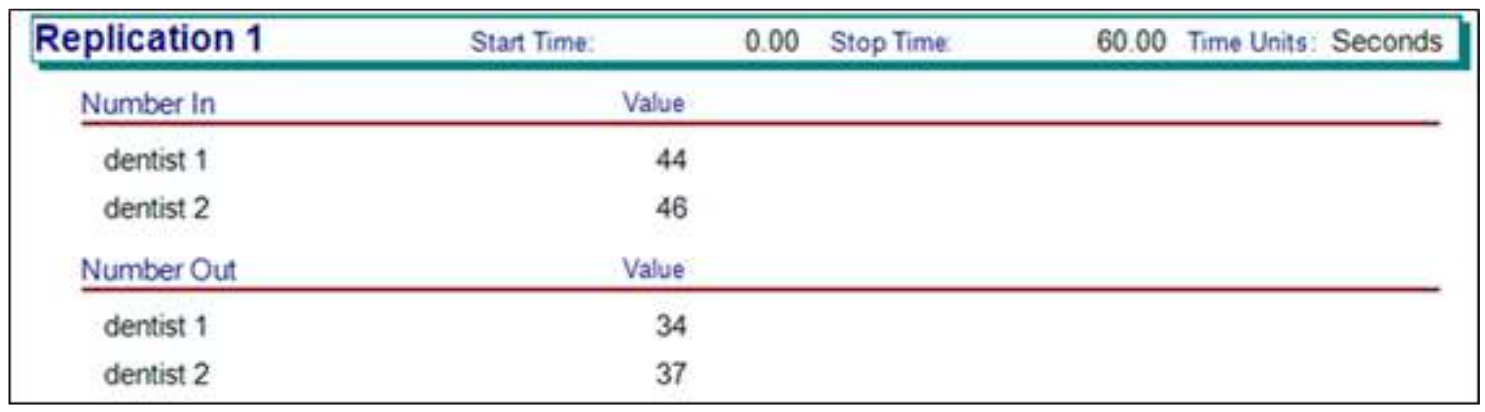

Figure (5). Result of patient in and out/model 1/replication 1.

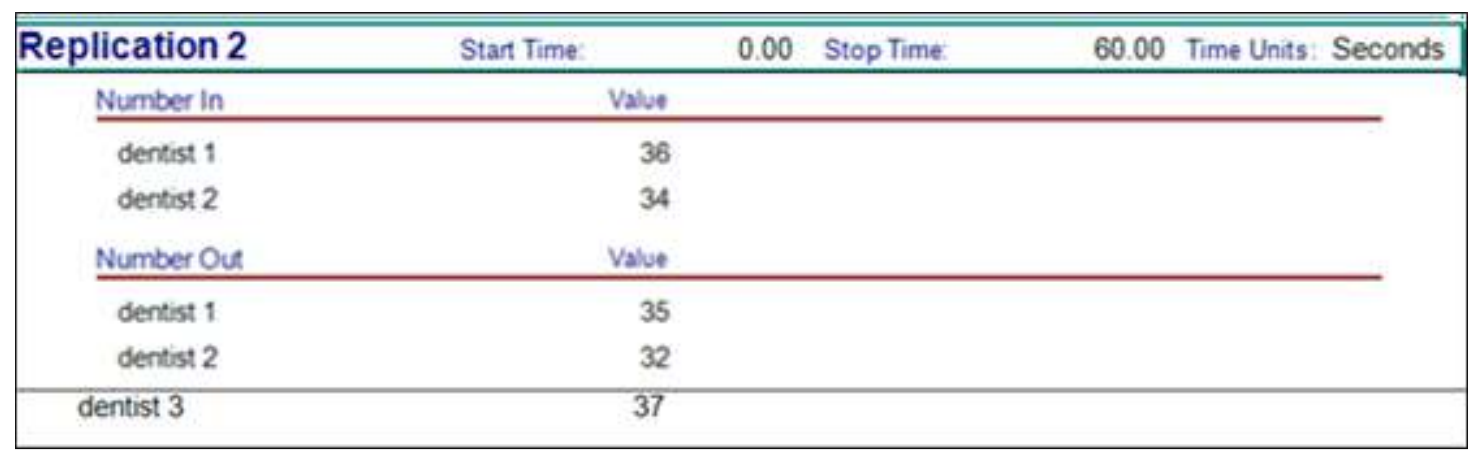

Figure (6). Result of patient in and out/ model 1/replication 2.

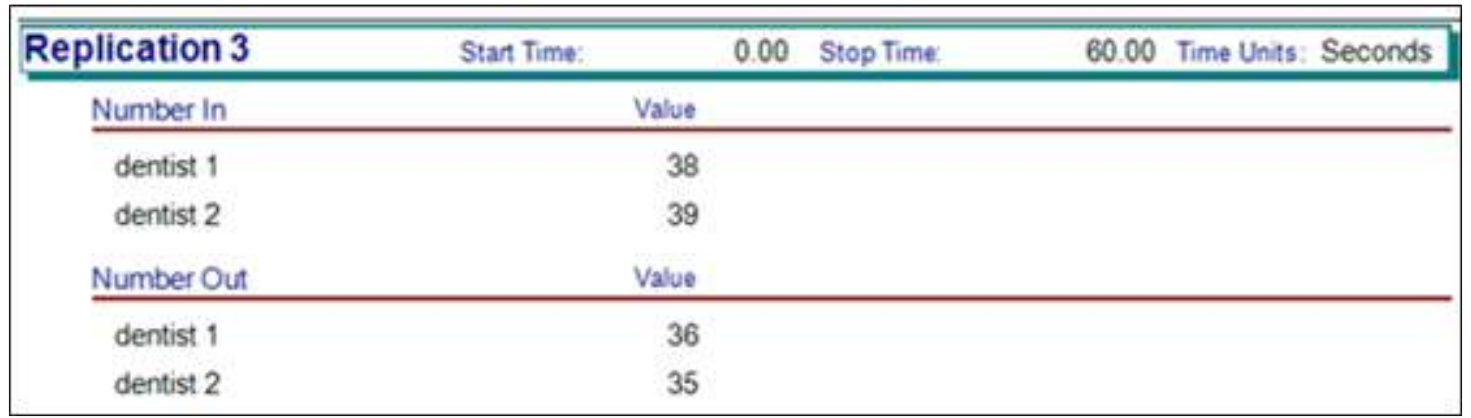

Figure (7). Result of patient in and out/ model 1/replication 3. 


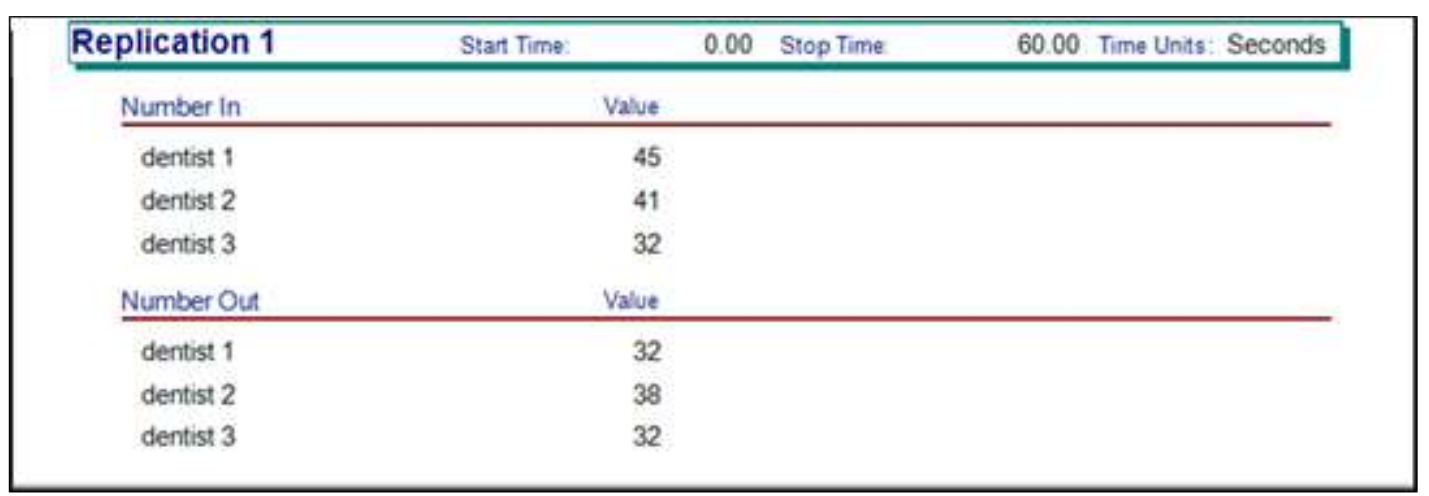

Figure (8). Result of patient in and out/ model 2/replication 1.

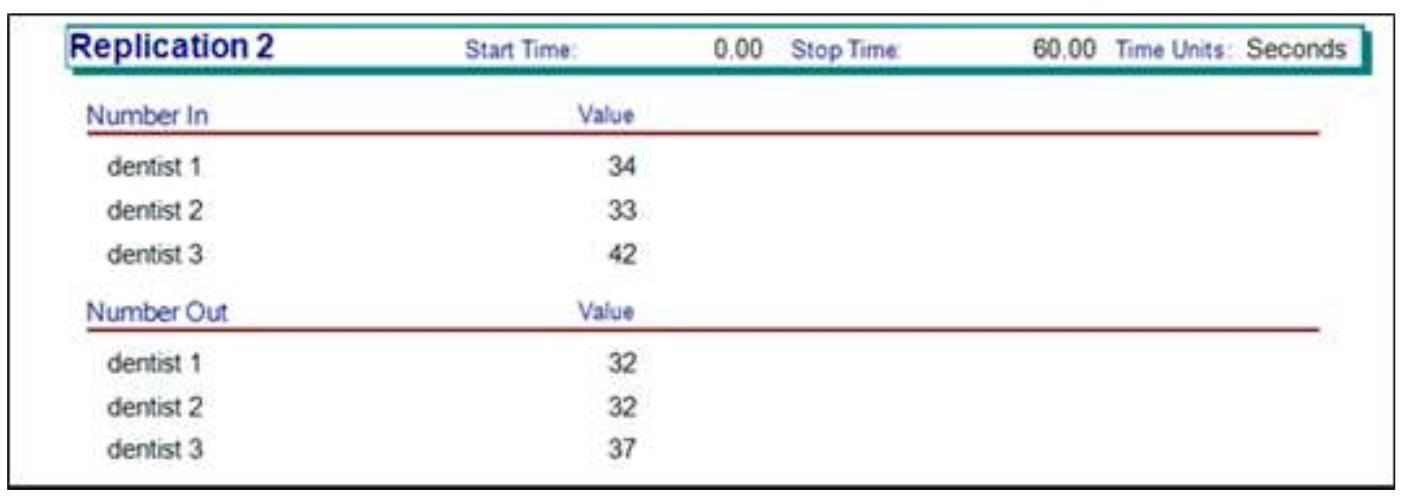

Figure (9). Result of patient in and out/ model 2/replication 2.

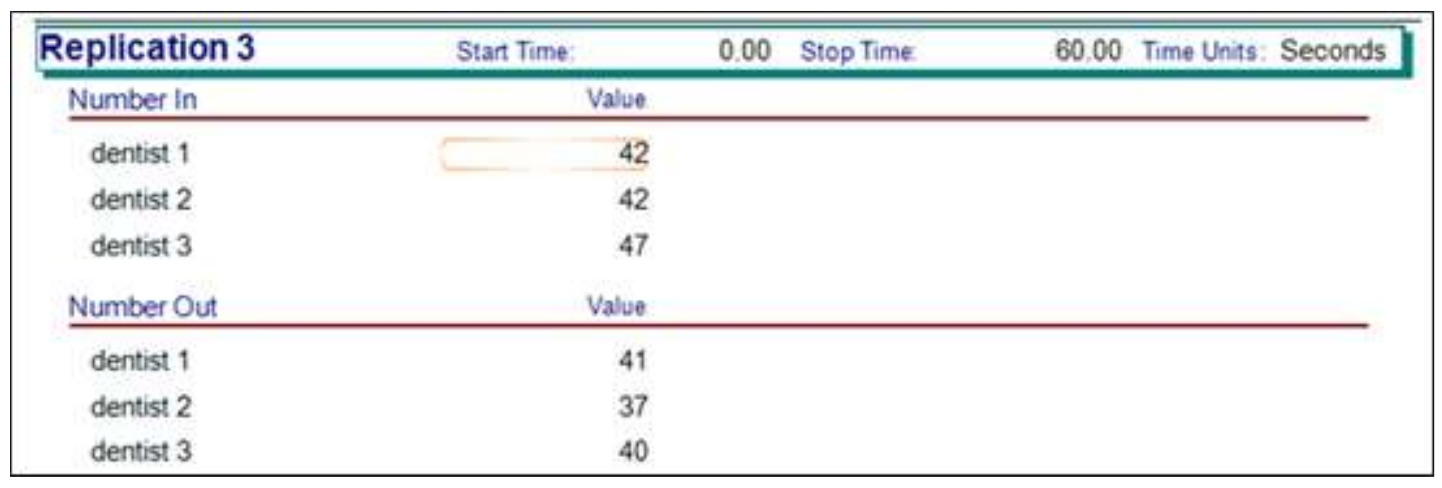

Figure (10). Result of patient in and out/ model 2/replication 3.

From Figures $(5,6,7,8,9, \& 10)$, we can conclude that, the increase of number of resources (which means here the third dentist) and making benefit of the experience of the third dentist will increase the number of patients who can check or be treated. Increasing the number of resources can improve the performance of other dentists with $7 \%$ which means an increase in the number of patients with 7\% those who are either be trated at the current clinic or sent to another outside clinic. A Graphical comparison of 3 replications that already conducted in Figures $(5,6$, $7,8,9, \& 10)$ is presented in Figure (11). 


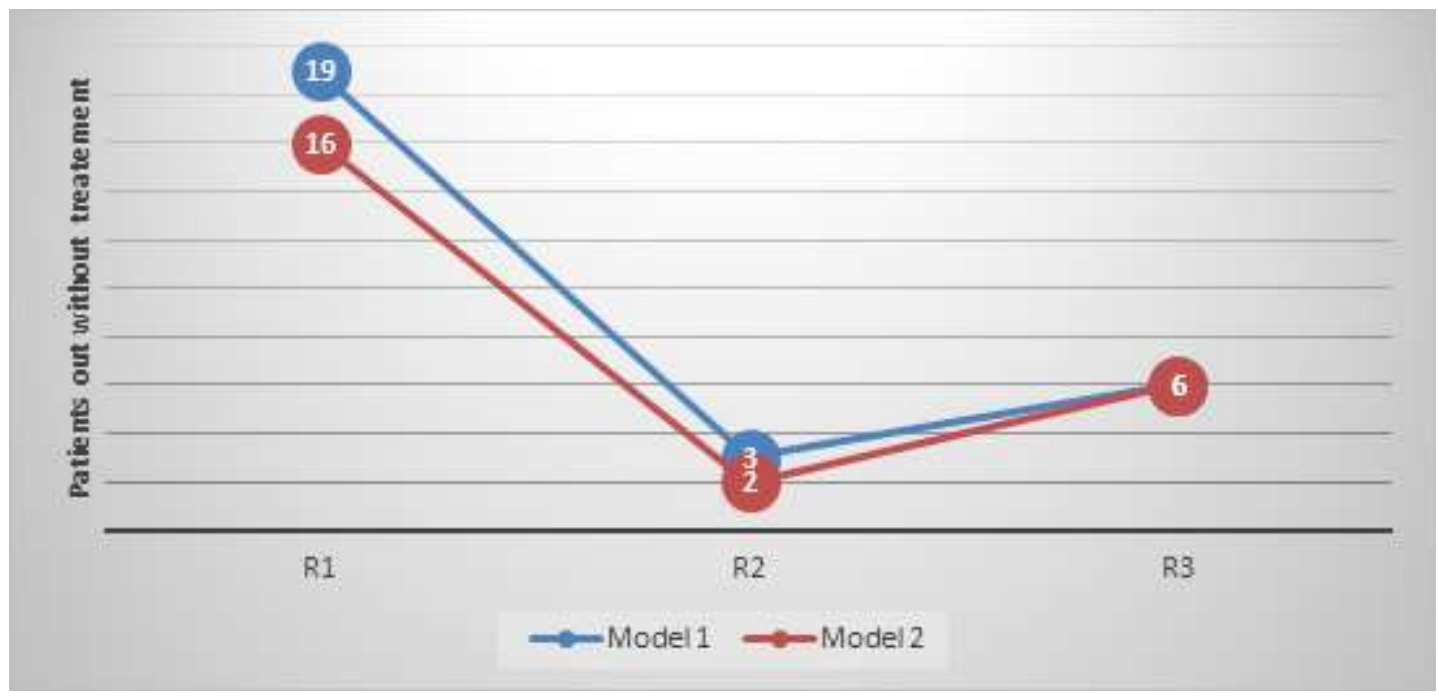

Figure (11). Performance of 3 replications for dentists.

The improvement in the performance of the dentists from model 2 is obvious in Figure (11) where the number of patients that could not take necessary treatment is 28 in model 1 while it is decreased to 24 in model 2 . These results are only for 3 replications as we have already mentioned, more experiments have been done for more than 3 replications. We are assuring that the results are improved more than $10 \%$.

\section{Conclusions}

Outpatients' clinics are one of the most important centers that people need, especially those with limited income, because these outpatient clinics are cheap or free in some countries. In Iraq, outpatients are generally considered cheap, but they lack resources and not properly utilized as the working doctors in relation to their number are unequal. This leads to a delay in the treatment of patients and a large waiting time, especially since the number of patients who resort to these clinics is very large. In this research, the work of a dental clinic in Iraq in Baghdad was improved by increasing the number of resources without need to spend money, which led to improving the performance of dentists and increasing the number of patients by $7 \%$ with the possibility of increasing the percentage to more than $10 \%$. On the other hand, these solutions may not be suitable for some other clinics due to lack of resources and funding.

Acknowledgement: We would like to thank all employers in the 7th April outpatient clinic in Baghdad/ Iraq to provide all the important and requested information to complete this research.

\section{References}

[1] J. A. Paul and L. Lin, "Models for improving patient throughput and waiting at hospital emergency departments," The Journal of emergency medicine, vol. 43, pp. 1119-1126, 2012.

[2] N. Hamza, M. A. Majid, K. Adam, and N. A. A. Bakar, "A Review on Simulation and Modelling for Patient Flow in Emergency Department," in IOP Conference Series: Materials Science and Engineering, 2019, p. 012037.

[3]P. Bocciarelli, A. D'Ambrogio, A. Giglio, and E. Paglia, "Simulation-based performance and reliability analysis of business processes," in Proceedings of the Winter Simulation Conference 2014, 2014, pp. 3012-3023.

[4]K. Silvester, R. Lendon, H. Bevan, R. Steyn, and P. Walley, "Reducing waiting times in the NHS: is lack of capacity the problem?," Clinician in Management, vol. 12, 2004.

[5] O. A. Arah, T. Custers, and N. S. Klazinga, "Updating the key dimensions of hospital performance: the move towards a theoretical framework," in Third WHO Workshop on Hospital Performance Measurement, Barcelona, 13-14 June 2003, 2003.

[6]S. Randall, T. Crawford, J. Currie, J. River, and V. Betihavas, "Impact of community based nurse-led clinics on patient outcomes, patient satisfaction, patient access and cost effectiveness: A systematic review," International journal of nursing studies, vol. 73, pp. 24-33, 2017. 
[7]D. L. White, "Operational planning and scheduling in the outpatient clinic environment," University of Cincinnati, 2010.

[8]R. Upadhyai, A. K. Jain, H. Roy, and V. Pant, "A Review of Healthcare Service Quality Dimensions and their Measurement," Journal of Health Management, vol. 21, pp. 102-127, 2019.

[9]I. Chishti, A. Basukoski, and T. Chaussalet, "Modeling and optimizing patient flows," in 8th Annual International Conference on ICT: Big Data, Cloud \& Security, 2017.

[10] A. Tayfur, M. Benjamin, " Simulation modeling and analysis with Arena", Elsevier, 2010.

[11] A. Erekat, A. Al-Shaebi, A. Alhaider, M. Khasawneh, and A. Khasawneh, "Optimizing Outpatient Access Center Staffing Using Discrete Event Simulation: A Case Study," in IIE Annual Conference. Proceedings, 2017, pp. 1918-1924.

[12] K. Kotiadis and A. A. Tako, "Facilitated post-model coding in discrete event simulation (DES): A case study in healthcare," European Journal of Operational Research, vol. 266, pp. 1120-1133, 2018.

[13] M. J. Glover, E. Jones, K. L. Masconi, M. J. Sweeting, S. G. Thompson, and S. Collaborators, "Discrete Event Simulation for Decision Modeling in Health Care: Lessons from Abdominal Aortic Aneurysm Screening," Medical Decision Making, vol. 38, pp. 439-451, 2018.

[14] N. Shukla, J. E. Keast, and D. Ceglarek, "Role activity diagram-based discrete event simulation model for healthcare service delivery processes," International Journal of Systems Science: Operations \& Logistics, vol. 4, pp. 68-83, 2017.

[15] J. L. Wiler, R. T. Griffey, and T. Olsen, "Review of modeling approaches for emergency department patient flow and crowding research," Academic Emergency Medicine, vol. 18, pp. 1371-1379, 2011.

[16] B. S. Onggo, N. Proudlove, S. D’Ambrogio, A. Calabrese, S. Bisogno, and N. Levialdi Ghiron, "A BPMN extension to support discrete-event simulation for healthcare applications: an explicit representation of queues, attributes and data-driven decision points," Journal of the operational research society, vol. 69, pp. 788-802, 2018. 